\title{
Sinunasale Symptome ANCA-assoziierter Vaskulitiden und deren Therapie mit Liposomen
}

\author{
Liposomal Local Therapy of Sinunasal Symptoms in ANCA Associated \\ Vasculitis
}

\author{
Autoren \\ B. Hofauer, M. Bas, U. Straßen, Y. Matsuba, N. Mansour, A. Knopf \\ Institut \\ Klinikum rechts der Isar, Hals-Nasen-Ohrenklinik und Poliklinik, München
}

Schlüsselwörter

- Granulomatose

- Morbus Wegener

- ANCA

- Churg-Strauss-Syndrom

- Liposomen

Key words

- granulomatosis

- Wegener's

- ANCA

- Churg-Strauss syndrome

- liposomes

eingereicht 02 . Oktober 2013

akzeptiert 05. März 2014

Bibliografie

DOI http://dx.doi.org/

10.1055/s-0034-1372588

Online-Publikation: 28.4 .2014

Laryngo-Rhino-Otol 2014; 93 :

461-466 @ Georg Thieme

Verlag KG Stuttgart · New York

ISSN 0935-8943

Korrespondenzadresse

Dr. Benedikt Hofauer

Klinikum rechts der Isar

Hals-Nasen-Ohrenklinik und

Poliklinik

Ismaningerstraße 22

81675 München

b.hofauer@lrz.tum.de

License terms

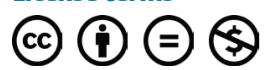

\section{Zusammenfassung}

\section{$\nabla$}

Hintergrund: Zu den ANCA-assoziierten Vaskulitiden (AAV) zählen die Granulomatose mit Polyangiitis (GPA), die eosinophile Granulomatose mit Polyangiitis (eGPA) und die Mikroskopische Polyangiitis (MPA). Eine Manifestation dieser Erkrankungen im HNO-Bereich ist Teil des typischen Beschwerdebildes. In einer Vielzahl der Fälle wird, meist auf der Grundlage von Organbeteiligungen, eine immunmodulatorische Therapie durchgeführt. Es lässt sich jedoch regelmäßig beobachten, dass allein dadurch eine nur unzureichende Linderung sinunasaler Symptome erzielt werden kann.

Material und Methoden: Zwischen Februar 2009 und November 2012 wurden 20 Patienten mit einer AAV in der Hals-Nasen-Ohrenklinik des Klinikums rechts der Isar diagnostiziert oder mit bekannter AAV zur Therapie überwiesen. Zur Erfassung des klinischen Beschwerdebildes wurden anerkannte Krankheitsaktivitätsindizes verwendet. Der Effekt einer Lokaltherapie mit Lipo- somen auf die sinunasale Symptomatik wurde über einen Zeitraum von 2 Monaten prospektiv ermittelt. Hierfür wurden sowohl standardisierte Fragebögen als auch visuelle Analogskalen verwendet.

Ergebnisse: Im beschriebenen Kollektiv zeigten sich bei $100 \%$ der Patienten Symptome im HNO-Bereich, alle Patienten wurden einer immunmodulatorischen Therapie zugeführt. Bei 61,1\% der Patienten zeigte sich hierdurch lediglich eine geringe bzw. keine Besserung der sinunasalen Symptome. Nach 2-monatiger Lokaltherapie mit Liposomen zeigten sich signifikante Verbesserungen sowohl der mittels visuellen Analogskalen erfassten subjektiven Beschwerden ( $p<0,001$ bis $p=0,014$ für die verschiedenen Symptome) als auch der mittels standardisierten Fragebögen ermittelten Werte $(p<0,001)$.

Schlussfolgerungen: Der lokaltherapeutische Einsatz von Liposomen eignet sich zur effektiven Behandlung sinunasaler Symptome bei Patienten mit AAV und stellt eine sinnvolle Erweiterung zur alleinigen systemischen Therapie dar.

\begin{tabular}{ll}
\hline Abkürzungen \\
AAV & ANCA-assoziierte Vaskulitis \\
ANCA & $\begin{array}{l}\text { Antineutrophilen cytoplasmatische } \\
\text { Antikörper }\end{array}$ \\
BVAS & Birmingham Vaskulitis Aktivitäts Score \\
CSS & Churg Strauss Syndrome \\
eGPA & eosinophile Granulomatose mit \\
& Polyangiitis \\
EULAR & European League Against Rheumatism \\
EUVAS & European Vasculitis Study Group \\
FFS & Five-Factors Score \\
GPA & Granulomatose mit Polyangiitis \\
MPA & Mikroskopische Polyangiitis \\
NAB & Nasenatmungsbehinderung \\
VAS & Visuelle Analogskala \\
VDI & Vasculitis Damage Index
\end{tabular}

\section{Einleitung \\ $\nabla$}

Zu den ANCA-assoziierten Vaskulitiden (AAV) zählen die Granulomatose mit Polyangiitis (GPA, ehemals Morbus Wegener), die eosinophile Granulomatose mit Polyangiitis (eGPA, ehemals Churg-Strauss-Syndrom) und die Mikroskopische Polyangiitis (MPA). Mit einer Inzidenz von 9,5 bis 16 auf eine Million Einwohner umfasst diese Gruppe die häufigsten Formen der primär systemischen Kleingefäßvaskulitiden, deren frühzeitige Diagnose und konsekutive Therapie von entscheidender Bedeutung ist, um möglichen lebensbedrohlichen Organschäden vorzubeugen [1]. Der Befall von Arteriolen, Venolen und Kapillaren kann sich in nahezu jedem Organsystem äußern. Eine nekrotisierende Glomerulonephritis 
und der Befall der unteren Atemwege treten regelhaft im Rahmen einer GPA/MPA auf. Der pulmonale Befall in Form eines Asthma bronchiales stellt die häufigste Manifestation der eGPA dar. Manifestationen im HNO-Bereich werden für AAV mit einer Häufigkeit von 35\% (MPA) bis 90\% (GPA) angegeben. Typischerweise wird für die GPA eine „ELK-Trias“ (ear, nose and throat/ lung/kidney) beschrieben [2]. Klinisch ist die GPA kaum von der MPA zu unterscheiden. Typische Symptome im HNO-Bereich reichen von nasalen Verborkungen, blutiger Rhinorrhoe, rezidivierenden Sinusitiden und Epistaxis bis hin zu Septumnekrosen und Sattelnasendeformitäten. Im Bereich der Ohren kann es zu rezidivierenden Otitiden und zu einer sensorineuralen Schwerhörigkeit kommen. Subglottische Stenosen können zu Stridor und lebensbedrohlicher Dyspnoe führen und auch von einer Mitbeteiligung der großen Kopfspeicheldrüsen inklusive Schädigung des N. facialis wird berichtet [3]. In etwa $50 \%$ aller Patienten mit einer eGPA kommt es zu einer allergischen Rhinitis mit Nasenatmungsbehinderung und chronisch polypöser Rhinosinusitis. Die GPA zeichnet sich durch das Vorliegen einer nekrotisierenden granulomatösen Entzündung ohne Nachweis asthmatischer Charakteristika aus. Im Falle der eGPA handelt es sich um eine nekrotisierende granulomatöse Entzündung in Verbindung mit Eosinophilie und Asthma bronchiale, wohingegen bei der MPA typischerweise weder granulomatöse Entzündungen noch ein Asthma zu finden sind [4]. Für die GPA wird darüber hinaus eine limitierte Form mit einem isolierten Befall der oberen Atemwege beschrieben, welche in $25 \%$ aller Fälle zu diagnostizieren ist [5,6]. Das Erkrankungsalter soll hier, entgegen des Beginns der generalisierten Form zwischen dem 40. und 55. Lebensjahr, deutlich früher sein [1]. Während ein positiver Nachweis antineutrophiler zytoplasmatischer Antikörper (ANCA) bei generalisierten Formen der GPA und MPA üblicherweise in 82-94\% möglich ist, gelingt dieser bei der limitierten Form deutlich seltener. Von der systemischen Ausprägung ist das männliche Geschlecht etwas häufiger betroffen, wohingegen die limitierte Form das weibliche Geschlecht häufiger zu betreffen scheint.

Die initiale immunmodulatorische Therapie sowohl der GPA, eGPA als auch der MPA beinhaltet in der Regel den Einsatz von Cyclophosphamid und Glukokortikoiden. Darüber hinaus kommen Rituximab und Methotrexat zum Einsatz. Der positive Effekt auf das Gesamtüberleben rechtfertigt den Einsatz dieser unter Umständen mit Nebenwirkungen verbundenen Therapie [7]. Seit dem Einsatz immunsupprimierender und -modulierender Therapeutika konnte die 5-Jahre-Überlebenszeit von anfangs annähernd 0 auf nun $90 \%$ verbessert werden [8]. Wiederholt lässt sich jedoch im klinischen Alltag beobachten, dass trotz aggressiver Systemtherapie die Symptomatik im Bereich der oberen Atemwege unverändert bleibt. Als mögliche Ursache wird eine bakterielle Übersiedlung mit konsekutiver Infekttriggerung des schwer zugänglichen Sinunasalsystems genannt, jedoch erbrachte der Einsatz lokaler antibiotikahaltiger Salben nicht den erwünschten Langzeiteffekt.

Bis zum heutigen Zeitpunkt liegen keinerlei Studien zur supportiven topischen Therapie sinunasaler Beschwerden ANCA-assoziierter Vaskulitiden vor. Allgemeine Behandlungsempfehlungen zum Einsatz bestimmter Lokaltherapeutika auf der Grundlage einer schonenden und effektiven Symptomkupierung liegen nicht vor. In der folgenden Arbeit wird der Effekt einer Lokaltherapie mit Liposomen auf die sinunasalen Symptome ANCA-assoziierter Vaskulitiden vorgestellt. Hierbei handelt es sich um in liposomaler Form vorliegende Phospholipide, welche, mittels eines Sprays appliziert, zur Stabilisierung der Lipidschicht und
Tab. 1 Mittels visueller Analogskala beurteilte sinunasale Beschwerden.

$$
\begin{aligned}
& \text { Sinunasale Beschwerden } \\
& \text { Borkige Rhinitis/Verkrustung } \\
& \text { Blutige Rhinorrhoe } \\
& \text { Nasenatmungsbehinderung } \\
& \text { Rezidivierende Epistaxis } \\
& \text { Hyposmie } \\
& \text { Rhinitis sicca } \\
& \text { Rezidivierende Sinusitis }
\end{aligned}
$$

somit zur Unterstützung der natürlichen Barrierefunktion der Schleimhäute beitragen sollen.

\section{Material und Methoden \\ $\nabla$}

\section{Patientenkollektiv}

Im Zeitraum zwischen Februar 2009 und November 2012 wurden insgesamt 20 Patienten mit einer AAV diagnostiziert oder mit bekannter AAV zur Therapie überwiesen. Die Diagnose folgte den aktuellen Empfehlungen der European Vasculitis Study Group (EUVAS) [9] und den Klassifikationskriterien des American College of Rheumatology (ACR) [10] sowie entsprechend der Definitionen der Chapel Hill Consensus Conference (CHCC) [11]. Das Durchschnittsalter zum Zeitpunkt der Diagnose, die Geschlechterverteilung, Krankheitsdauer sowie systemische und lokale Symptome wurden durch Auswertung der Krankenakten ermittelt. Die Krankheitsaktivität zum Zeitpunkt der Diagnose wurde bei Patienten mit GPA oder MPA mithilfe des Birmingham-Vaskulitis-Aktivitäts-Scores (BVAS) [12], des für Patienten mit GPA modifizierten BVAS (BVAS/WG) [13] und des Krankheitsaktivitäts-Index für GPA der deutschen Gesellschaft für Rheumatologie bestimmt. Zur Bestimmung des durch die Vaskulitis verursachten organischen Schäden wurde der Vasculitis Damage Index (VDI) [14] angewandt. Zur Bestimmung der Krankheitsaktivität der eGPA kamen BVAS, VDI und der Five-Factors Score (FSS) [15] zum Einsatz.

\section{Behandlungsablauf}

In die vorliegende Studie wurden 18 Patienten eingeschlossen, 15 Patienten mit einer GPA, 2 Patienten mit einer eGPA und ein Fall einer MPA, die trotz suffizienter Therapie der systemischen Manifestation eine lokale Manifestation der Kopf-Hals-Region erlitten. 2 Patienten, die zu keinem Zeitpunkt im Verlauf ihrer Krankengeschichte sinunasale Symptome angaben und deren Spiegelbefund sich als unauffällig darstellte, wurden nicht in die weiteren Untersuchungen eingeschlossen. Vor Beginn der nasalen Lokaltherapie mit Liposomen (LipoNasal@, Optima Pharmazeutische $\mathrm{GmbH}$, Wang, Deutschland) wurden die HNO-Symptome der jeweiligen standardisierten Fragebögen (BVAS und BVAS/ WG für GPA/MPA; BVAS für eGPA) erneut erhoben. Die subjektiven sinunasalen Beschwerden jedes Patienten wurden unter Zuhilfenahme visueller Analogskalen (VAS) bewertet. Hierbei wurden subjektive Einschätzungen zu den in $\bullet$ Tab. 1 aufgezählten Beschwerden anhand einer Skala von 1 (=keine Beschwerden/ trifft nicht zu) bis 10 (=maximale Symptomausprägung) eingestuft. Zusätzlich wurden die HNO-Symptome eines kürzlich veröffentlichten Krankheitsaktivitätsindex zur Beurteilung der HNO-Beteiligung einer GPA bestimmt (ENT/GPA DAS) [16]. Die Patienten wurden in den korrekten Gebrauch des liposomalen Pflegeprodukts eingewiesen. Es sollte eine beidseitige, 6-mal 


$\begin{array}{llll}\begin{array}{l}\text { Zeitpunkt Parameter } \\ \text { BVAS }\end{array} & \begin{array}{l}\text { Erstdiagnose } \\ \text { GPA/MPA/EGPA } \\ \text { BVAS }\end{array} & \begin{array}{l}\text { Therapiebeginn } \\ \text { HNO-Symptome }\end{array} & \text { Nach } 2 \text { Monaten } \\ \begin{array}{l}\text { BVAS/WG } \\ \text { BVAS/WG }\end{array} & \text { GPA/MPA } & \text { GPA/MPA/EGPA } & \\ \text { HNO-Symptome } & \text { GPA/MPA } & \text { GPA/MPA } & \text { GPA/MPA } \\ \text { Krankheitsaktivitäts-index für GPA } & \text { GPA/MPA/EGPA } & & \\ \text { VDI } & \text { GPA/MPA/EGPA } & & \\ \text { FFS } & \text { EGPA } & \text { GPA/MPA } & \text { GPA/MPA } \\ \text { ENT/GPA DAS } & & \text { GPA/MPA/EGPA } & \text { GPA/MPA/EGPA } \\ \text { VAS } & & \end{array}$

Tab. 2 Übersicht zu erhobenen Parametern bei Erstdiagnose, Therapiebeginn und nach 2-monatiger Therapie.

BVAS Birmingham-Vaskulitis-Aktivitäts-Score

BVAS/WG Birmingham-Vaskulitis-Aktivitäts-Score modifiziert für GPA

VDI Vaskulitis Damage Index

FFS Five-Factors Score

ENT/GPA DAS Disease Activity Score for ENT Involvement in GPA

VAS Visuelle Analogskala

tägliche Anwendung erfolgen und auf den Gebrauch weiterer nasal angewendeter Produkte verzichtet werden. Es wurde darauf geachtet, dass zu Beginn und während des Behandlungszeitraumes keine Änderungen der systemischen Therapie der AAV vorgenommen wurde. Nach 2-monatiger konsequenter liposomaler Lokaltherapie erfolgte eine erneute Evaluation dieser erhobenen Parameter. In $\bullet$ Tab. $\mathbf{2}$ wird dargestellt, zu welchem Zeitpunkt welche Parameter erhoben wurden. Zur statistischen Auswertung wurde SPSS 16.0 verwendet.

\section{Ergebnisse}

\section{Epidemiologie des Patientenkollektivs}

Das durchschnittliche Patientenalter betrug 57,3 Jahre (SD: 12,9 Jahre) und bei 12 der 18 Patienten handelte es sich um Frauen. Die durchschnittliche Erkrankungsdauer betrug 61,2 Monate (SD: 73 Monate). Zum Zeitpunkt der Diagnosestellung zeigten 10 Patienten Leitsymptome im HNO-Bereich, in Anamnese und klinischer Untersuchung konnten jedoch in allen 18 Patienten unterschiedliche Manifestationen ANCA-assoziierter Vaskulitiden im HNO-Bereich nachgewiesen werden. Im Rahmen der Erstdiagnose wurde ein Mittelwert des BVAS-Scores von 16,7 (SD: 7,9) (maximal 63 Punkte möglich) erreicht. In der Auswertung des für GPA angepassten BVAS/WG-Scores zeigte sich ein Mittelwert der Major-Symptome von 1,3 (SD: 1,3) und der Minor-Symptome von 5,0 (SD: 2,4). Folglich konnte in 10 der 15 Patienten mit GPA die Erkrankung als schwerwiegend eingestuft werden, in 5 Fällen als limitiert. Der Krankheitsaktivitäts-Index für GPA ergab für das beschriebene Patientenkollektiv einen Mittelwert von 15,5 (SD: 9,1) (maximal 127 Punkte möglich). Der verwendete VDI zur Graduierung der organischen Schäden ergab einen Mittelwert von 4,9 (SD: 2,7) (maximal 64 Punkte möglich). Der FFS zur Bestimmung der Aktivität der eGPA, welcher auch als Prognoseparameter dient, ergab im Mittelwert einen Score von 0 (maximal 5 Punkte möglich).

Alle Patienten wurden im Rahmen ihrer AAV einer immunmodulatorischen Therapie zugeführt, eine vollständige Remission sinunasaler Beschwerden wurde von keinem Patienten angegeben, 3 Patienten gaben einen deutlichen, 4 Patienten einen teilweisen und 11 Patienten einen geringen bzw. keinen Effekt der immunmodulatorischen Therapie hinsichtlich sinunasaler Beschwerden an ( $\bullet$ Abb. 1).

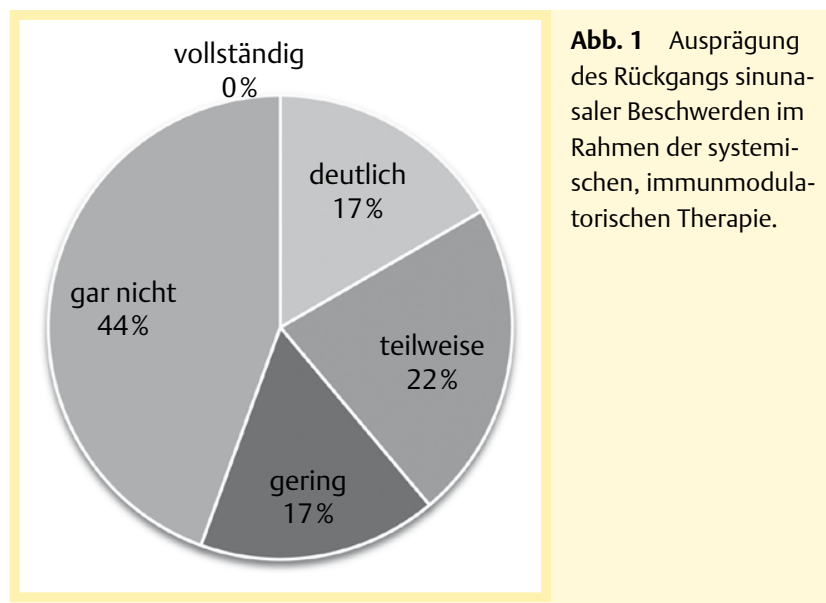

Effekt der liposomalen Lokaltherapie auf die sinunasalen Beschwerden

Die Mittelwerte der mithilfe der VAS angegebenen nasalen Verborkung betrug 5,8 (SD: 2,7), der blutigen Rhinorrhoe 5,3 (SD: 3,4 ), der Nasenatmungsbehinderung 5,8 (SD: 2,8), der rezidivierenden Epistaxis 4,1 (SD: 3,7), der Hyposmie 4,6 (SD: 3,3), der Rhinitis sicca 6,1 (SD: 2,9) und der rezidivierenden Sinusitiden 2,7 (SD: 1,9). Die Mittelwerte nach Durchführung der liposomalen Lokaltherapie betrugen für den Grad der nasalen Verborkung 3,0 (SD: 1,8), der blutigen Rhinorrhoe 2,6 (SD: 2,1), der Nasenatmungsbehinderung 2,8 (SD: 1,7), der rezidivierenden Epistaxis 1,7 (SD: 1,3), der Hyposmie 3,7 (SD: 3,4), der Rhinitis sicca 2,9 (SD: 2,1) und der rezidivierenden Sinusitiden 1,4 (SD: $1,0)$. Durchschnittlich trat durch die liposomale Pflege eine Verbesserung von 2,32 (Min: 0,8; Max: 3,2) Punkten der VAS ein. Der Rückgang der nasalen Verborkung, der blutigen Rhinorrhoe, der Nasenatmungsbehinderung sowie der Rhinitis sicca erreichte jeweils eine Signifikanz von $\mathrm{p}<0,001$. Die rezidivierenden Sinusitiden verbesserten sich mit einer Signifikanz von $p=0,001$, rezidivierende Epistaxis mit einer Signifikanz von $p=0,005$ und die Hyposmie verbesserte sich mit einem Signifikanzgrad von $\mathrm{p}=0,014$ ( $\bullet$ Abb. 2).

Vor Beginn der nasalen Lokaltherapie mit Liposomen wurden die HNO-Symptome der verwendeten standardisierten Fragebögen erneut evaluiert. Für die HNO-Rubrik des BVAS ergab sich ein Mittelwert von 9,6 Punkten (SD 3,9) vor, und von 2,3 Punkten 


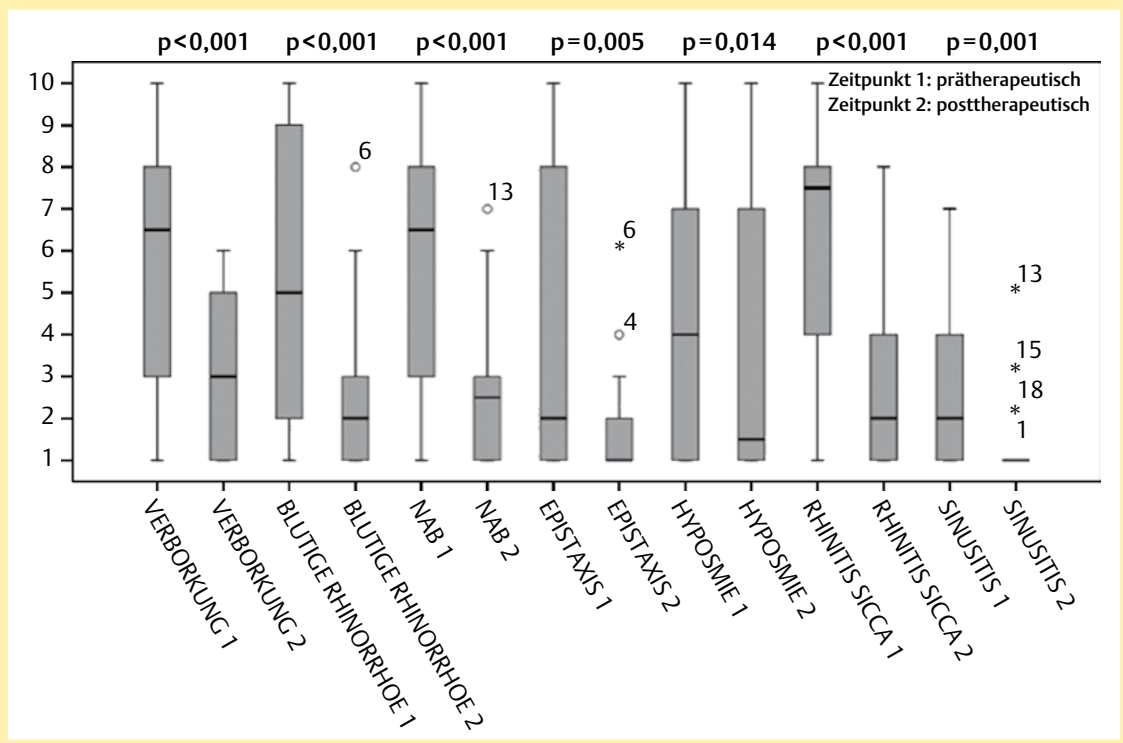

Abb. 2 Effekt der liposomalen Lokaltherapie auf die mittels VAS erfassten subjektiven sinunasalen Beschwerden.

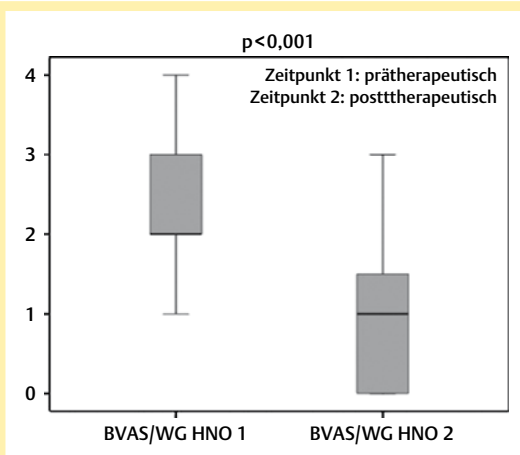

Abb. 3 Effekt der liposomalen Lokaltherapie auf die HNO-Symptome des Birmingham-VaskulitisAktivitäts-Scores.

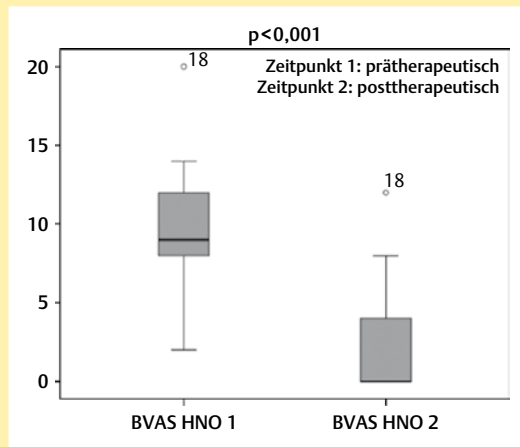

Abb. 4 Effekt der liposomalen Lokaltherapie auf die HNO-Symptome des für GPA modifizierten Birmingham-VaskulitisAktivitäts-Scores.

(SD 3,4) während der Therapie und somit ein Rückgang um durchschnittlich 7,2 Punkte (SD 4,1; p<0,001) ( $\bullet$ Abb. 3). Hinsichtlich der HNO-Symptome des BVAS/WG zeigte sich ein ähnliches Bild. Auch hier resultierte die Lokaltherapie in einer Verbesserung von anfangs 2,5 (SD: 0,9) um 1,6 Punkte (SD: 0,8) auf 0,9 (SD: 0,9; p<0,001) ( $\bullet$ Abb. 4). Dieser Effekt konnte auch in dem erst kürzlich veröffentlichten Krankheitsaktivitätsindex nachvollzogen werden. Von den $6 \mathrm{zu}$ beurteilenden Kriterien wurden vor Beginn der Lokaltherapie im Mittel 2,0 (SD: 0,6) als positiv gewertet. Durch den Einsatz nasaler Liposomen besserte sich auch dieser Index um 1,2 (SD: 0,7), sodass im Verlauf nur noch 0,8 (SD: 0,8) Kriterien als positiv gewertet wurden $(\mathrm{p}<0,001)$.

Nebenwirkungen wurden während der gesamten Therapiedauer nicht beobachtet.

\section{Diskussion}

Diese prospektiv durchgeführte Studie diente der erstmaligen Untersuchung eines Lokaltherapeutikums zur Verbesserung des sinunasalen Beschwerdebildes bei Patienten mit AAV. In den vergangenen Jahren konnten die immunmodulatorischen Regime zur Therapie systemischer und potentiell lebensbedrohlicher Beschwerden, die sich wiederum in eine Induktionstherapie und eine Erhaltungstherapie unterteilen lassen, stetig verbessert werden und so die Mortalitätsrate deutlich reduziert werden. Diese Entwicklung veränderte den Verlauf der AAV hin zu einem chronischen Beschwerdebild und steigerte somit den Bedarf einer zusätzlichen supportiven Therapie lokaler, nicht lebensbedrohlicher, jedoch die Lebensqualität deutlich einschränkender Symptome. Es zeigt sich wiederholt, dass durch eine reine Systemtherapie sinunasale Symptome nicht vollständig bzw. ausreichend gelindert werden können. Darüber hinaus wird diskutiert, ob persistierende nasale Verborkungen, Keimbesiedelungen und rezidivierende Infekte eine erhöhte Rückfallneigung bedingen [17]. Somit ergäben sich, neben einer reinen Verbesserung der Lebensqualität des Patienten, durchaus auch prognostische Argumente zur Durchführung einer effektiven Lokaltherapie.

Neben den bereits beschriebenen, endoskopisch sichtbaren sinunasalen Folgen im Rahmen einer AAV kommt es auch zu makroskopisch nicht nachzuvollziehenden Veränderungen. Ullrich et al. stellten in ihren Untersuchungen der Zilientätigkeit des respiratorischen Epithels eine deutlich reduzierte Schlagfrequenz fest [18]. Wenngleich es bisher unklar ist, ob diese Veränderung auf die Erkrankung selbst oder auf unerwünschte Arzneimittelwirkungen im Rahmen der Systemtherapie zurückzuführen ist, stellt dies eine deutliche Einschränkung des Abwehrsystems der oberen Atemwege dar. Auch Untersuchungen des molekularen Expressionsmusters der nasalen Schleimhaut im Rahmen antibakterieller Reaktionen zeigten Unterschiede zwischen Patienten mit GPA und chronischer Rhinosinusitis auf. Es wird vermutet, dass hiermit erstmals möglicherweise krankheitsrelevante mRNA-Expressionsmuster nachgewiesen werden konnten und dies, in Verbindung mit einem exogenen Trigger, die ätiologische Grundlage dieser Erkrankung bilden könnte [17]. Als möglicher exogener Trigger wird abermals die Kolonisation mit Staphylococcus aureus diskutiert [19]. Im Vergleich zu verschiedenen 
Kontrollgruppen zeigten Patienten mit GPA signifikant höhere nasale Kolonisationsraten von bis zu 72\% [20]. In der Literatur findet man wiederholt die Beobachtung, dass ein sinunasaler Befall mit Staphylococcus aureus mit einer erhöhten Rezidivneigung verbunden ist $[6,17,21]$. Bis dato lässt sich dieser Zusammenhang, bzw. inwiefern sich die beschriebenen Veränderungen gegenseitig beeinflussen, nicht ausreichend erklären, jedoch werden, auch unabhängig von einer Kolonisation mit S. aureus, Ergebnisse publiziert, die eine erhöhte Rezidivrate in Patientenkollektiven mit HNO-Beteiligung angeben als ohne HNO-Beteiligung. Martinez Del Pero et al. beschreibt in einem Patientenkollektiv von 138 Patienten mit GPA eine signifikant $(p=0,001)$ erhöhte Rezidivrate in der Subpopulation mit HNO-Beteiligung, welche ebenfalls in einem signifikantem Maße $(p=0,004)$ wiederum den HNO-Bereich betraf [7].

Viele dieser Beobachtungen lassen sich derzeit noch nicht ausreichend erklären und bedürfen weiterer Untersuchungen, um sich schlüssig in das Erkrankungsbild der GPA oder AAV einzufügen. Es lässt sich jedoch nicht von der Hand weisen, dass der Effekt einer effektiven topischen Therapie über eine reine Verbesserung der lokalen Symptome und damit der Lebensqualität auch eventuell Einfluss auf den weiteren Krankheitsverlauf des Patienten haben kann. Aktuell stehen Patienten und Arzt Produkte mit unterschiedlichen Inhalts- und Wirkstoffen zur Verfügung um, in Verbindung mit einer intensiven Nasentoilette, je nach anatomischen Verhältnissen entweder durch den Patienten selbst oder durch den HNO-Arzt durchgeführt, die Lokalsituation zu verbessern [21]. Die Empfehlungen reichen von topischen Antibiotika bis hin zu Meersalz-Nasenspülungen versetzt mit Natriumbikarbonat. Diese Empfehlungen beruhen größtenteils jedoch auf Expertenmeinungen. Aus diesem Grund entschlossen wir uns, ein in der Lokaltherapie des Sjögren-Syndrom bereits erfolgreich eingesetztes Lokaltherapeutikum zur Linderung sinunasaler Symptome ANCA-assoziierter Vaskulitiden einzusetzen [22]. Das in dieser Studie verwendete Präparat enthält Liposomen (Phospholipiddoppelschichtvesikel), die zu einem großen Anteil (75\%) den natürlichen nasalen Surfactant bilden. Durch die Substitution dieser Phospholipide soll der natürliche Surfactant stabilisiert werden und die natürliche Barrierefunktion unterstützt werden.

Nach Beendigung des 2-monatigen Untersuchungszeitraums zeigten sich sowohl in der Auswertung der VAS, als auch in den seitens der EUVAS zur Durchführung klinischer Studien empfohlenen standardisierten Fragebögen signifikante Verbesserungen. Basierend auf diese Untersuchungsergebnisse kann erstmals eine Empfehlung zu einer bestimmten Lokaltherapie ausgesprochen werden. Darüber hinaus steht nun für weitere zukünftige Studien von topischen Therapiemöglichkeiten ein entsprechend den aktuellen Standards validiertes Produkt für Kontrollgruppen zur Verfügung. Zweifelsohne müssen nun Untersuchungen des Einflusses von Liposomen auf die natürliche Schutzfunktion des nasalen Epithels folgen. Für die vorliegende Studie sind Limitationen des Studiendesigns zu berücksichtigen. Der leicht zu identifizierende Eigengeruch, der durch den wesentlichen liposomalen Bestandteil des verwendeten Produkts erzeugt wird, erschwerte die Durchführung Plazebo kontrollierter Studien oder eines Crossover-Designs nachhaltig.

Zusammenfassend lässt sich sagen, dass sich der Einsatz einer supportiven Lokaltherapie mit Liposomen zur effektiven Behandlung sinunasaler Symptome bei Patienten mit AAV eignet und eine sinnvolle Ergänzung zu systemischen Therapiemaßnahmen darstellt.

\section{Anmerkungen}

\section{$\nabla$}

Die zur Durchführung dieser Studie erforderlichen liposomalen Pflegeprodukte wurden dankenswerter Weise von der Firma Optima unentgeltlich zur Verfügung gestellt. Die Firma nahm keinen Einfluss auf die Durchführung der Studie und war weder in der Datensammlung, noch an der anschließenden Auswertung beteiligt.

\section{Abstract}

\section{Liposomal Local Therapy of Sinunasal Symptoms in ANCA Associated Vasculitis \\ $\nabla$}

Background: GPA, eGPA and MPA constitute the group of AAV. ENT manifestations are part of the typical clinical picture of these diseases. Usually, patients are treated with systemic immunomodulatory drugs, mostly based on organ affection. In clinical routine, an insufficient decrease of sinunasal manifestations during a solely systemic therapeutic concept can repeatedly be observed.

Material and Methods: Between February 2009 and November 2012, 20 patients with AAV were diagnosed in or referred to our department for further treatment. Clinical symptoms and manifestations were measured by the use of international accepted activity scores. The effect of a local therapy with liposomes for a period of 2 months on sinunasal symptoms was prospectively evaluated by using visual analogue scales and standardized questionnaires.

Results: Within the described collective $100 \%$ of patients did show ENT-symptoms at the time of initial diagnosis. Every patient did receive immunomodulatory therapy, but in $61.1 \%$ of cases there was just slight or no improvement on sinunasal symptoms. After a 2-month period of liposomal local therapy, a significant reduction of sinunasal complaints could be observed, both evaluated via visual analogue scales $(\mathrm{p}<0.001$ to $\mathrm{p}=0.014$, depending on the evaluated symptom) and standardized questionnaires $(\mathrm{p}<0.001)$.

Conclusions: The local application of liposomes in addition to a systemic therapy is effective in alleviating sinunasal manifestations in patients with AAV.

Interessenkonflikt: Kein Interessenkonflikt angegeben.

\section{Literatur}

1 Reinhold-Keller E, Zeidler A, Gutfleisch J, Peter HH, Raspe HH, Gross WL. Giant cell arteritis is more prevalent in urban than in rural populations: Results of an epidemiological study of primary systemic vasculitides in Germany. Rheumatology (Oxford) 2000; 39: 1396-1402

2 Trimarchi M, Sinico RA, Teggi R, Bussi M, Specks U, Meroni PL. Otorhinolaryngological manifestations in granulomatosis with polyangiitis (wegener's). Autoimmun Rev 2013; 12: 501-505

3 Barrett AW. Wegener's granulomatosis of the major salivary glands. J Oral Pathol Med 2012; 41: 721-727

4 Jennette JC, Falk RJ. Small-vessel vasculitis. N Engl J Med 1997; 337: $1512-1523$

5 Stone JH. Limited versus severe wegener's granulomatosis: Baseline data on patients in the wegener's granulomatosis etanercept trial. Arthritis Rheum 2003; 48: 2299-2309

6 Gottschlich S, Ambrosch P, Kramkowski D, Laudien M, Buchelt T, Gross $W L$, Hellmich $B$. Head and neck manifestations of wegener's granulomatosis. Rhinology 2006; 44: 227-233 
7 Martinez Del Pero M, Walsh M, Luqmani R, Flossmann O, Mukhtyar C, Jani $P$, Rasmussen $N$, Jayne $D$. Long-term damage to the ent system in wegener's granulomatosis. Eur Arch Otorhinolaryngol 2011; 268: 733-739

8 Reinhold-Keller E, Beuge N, Latza U, De Groot K, Rudert H, Nolle B, Heller $M$, Gross WL. An interdisciplinary approach to the care of patients with wegener's granulomatosis: Long-term outcome in 155 patients. Arthritis Rheum 2000; 43: 1021-1032

9 Hellmich B, Flossmann O, Gross WL, Bacon P, Cohen-Tervaert JW, Guillevin L, Jayne D, Mahr A, Merkel PA, Raspe H, Scott DG, Witter J, Yazici H, Luqmani RA. Eular recommendations for conducting clinical studies and/or clinical trials in systemic vasculitis: Focus on anti-neutrophil cytoplasm antibody-associated vasculitis. Ann Rheum Dis 2007; 66: 605-617

10 Fries JF, Hunder GG, Bloch DA, Michel BA, Arend WP, Calabrese LH, Fauci $A S$, Leavitt RY, Lie JT, Lightfoot RWJ. The american college of rheumatology 1990 criteria for the classification of vasculitis. Summary. Arthritis Rheum 1990; 33: 1135-1136

11 Jennette JC, Falk RJ, Andrassy K, Bacon PA, Churg J, Gross WL, Hagen EC, Hoffman GS, Hunder GG, Kallenberg CG. Nomenclature of systemic vasculitides. Proposal of an international consensus conference. Arthritis Rheum 1994; 37: 187-192

12 Luqmani RA, Bacon PA, Moots RJ, Janssen BA, Pall A, Emery P, Savage $C$, Adu $D$. Birmingham vasculitis activity score (bvas) in systemic necrotizing vasculitis. QJM 1994; 87: 671-678

13 Stone JH, Hoffman GS, Merkel PA, Min YI, Uhlfelder ML, Hellmann DB, Specks U, Allen NB, Davis JC, Spiera RF, Calabrese LH, Wigley FM, Maiden N, Valente RM, Niles JL, Fye KH, Mccune JW, St Clair EW, Luqmani $R A$. A disease-specific activity index for wegener's granulomatosis: Modification of the birmingham vasculitis activity score. International network for the study of the systemic vasculitides (inssys). Arthritis Rheum 2001; 44: 912-920

14 Exley AR, Bacon PA, Luqmani RA, Kitas GD, Gordon C, Savage CO, Adu $D$. Development and initial validation of the vasculitis damage index for the standardized clinical assessment of damage in the systemic vasculitides. Arthritis Rheum 1997; 40: 371-380
15 Guillevin L, Lhote F, Gayraud M, Cohen P, Jarrousse B, Lortholary $O$, Thibult $N$, Casassus $P$. Prognostic factors in polyarteritis nodosa and churg-strauss syndrome. A prospective study in 342 patients. Medicine (Baltimore) 1996; 75: 17-28

16 Del Pero MM, Chaudhry A, Rasmussen N, Jani P, Jayne D. A disease activity score for ent involvement in granulomatosis with polyangiitis (wegener's). Laryngoscope 2013; 123: 622-628

17 Laudien M, Hasler R, Wohlers J, Bock J, Lipinski S, Bremer L, Podschun R, Ambrosch $P$, Lamprecht $P$, Rosenstiel $P$, Till A. Molecular signatures of a disturbed nasal barrier function in the primary tissue of wegener's granulomatosis. Mucosal Immunol 2011; 4: 564-573

18 Ullrich S, Gustke H, Lamprecht P, Gross WL, Schumacher U, Ambrosch $P$, Laudien $M$. Severe impaired respiratory ciliary function in wegener granulomatosis. Ann Rheum Dis 2009; 68: 1067-1071

19 Brons RH, Bakker HI, Van Wijk RT, Van Dijk NW, Muller Kobold AC, Limburg PC, Manson WL, Kallenberg CG, Tervaert JW. Staphylococcal acid phosphatase binds to endothelial cells via charge interaction; a pathogenic role in wegener's granulomatosis? Clin Exp Immunol 2000; 119: 566-573

20 Laudien M, Gadola SD, Podschun R, Hedderich J, Paulsen J, ReinholdKeller E, Csernok E, Ambrosch P, Hellmich B, Moosig F, Gross WL, Sahly $H$, Lamprecht $P$. Nasal carriage of staphylococcus aureus and endonasal activity in wegener $s$ granulomatosis as compared to rheumatoid arthritis and chronic rhinosinusitis with nasal polyps. Clin Exp Rheumatol 2010; 28: 51-55

21 Rasmussen $N$. Management of the ear, nose, and throat manifestations of wegener granulomatosis: An otorhinolaryngologist's perspective. Curr Opin Rheumatol 2001; 13: 3-11

22 Hofauer B, Bas M, Manour N, Knopf A. Liposomal local therapy as treatment for sicca symptoms in patients with primary sjogren's syndrome. HNO 2013 